

\title{
Ultraviolet laser deposition of graphene thin films without catalytic layers
}

S. R. Sarath Kumar and H. N. Alshareef

Citation: Applied Physics Letters 102, 012110 (2013); doi: 10.1063/1.4773987

View online: http://dx.doi.org/10.1063/1.4773987

View Table of Contents: http://scitation.aip.org/content/aip/journal/apl/102/1?ver=pdfcov

Published by the AIP Publishing

\section{Articles you may be interested in}

Temperature dependent dielectric function in the near-infrared to vacuum-ultraviolet ultraviolet spectral range of alumina and yttria stabilized zirconia thin films

J. Appl. Phys. 114, 223509 (2013); 10.1063/1.4844515

Femtosecond laser fabrication of micro and nano-disks in single layer graphene using vortex Bessel beams Appl. Phys. Lett. 103, 241111 (2013); 10.1063/1.4846415

Photoexpansion and nano-lenslet formation in amorphous As2S3 thin films by $800 \mathrm{~nm}$ femtosecond laser irradiation

J. Appl. Phys. 112, 033105 (2012); 10.1063/1.4745021

Nanostructured europium oxide thin films deposited by pulsed laser ablation of a metallic target in a He buffer atmosphere

J. Vac. Sci. Technol. A 28, 1092 (2010); 10.1116/1.3457784

Synthesis and structural, electrochromic characterization of pulsed laser deposited vanadium oxide thin films J. Vac. Sci. Technol. A 19, 887 (2001); 10.1116/1.1359533

You don't

still use this

cell phone

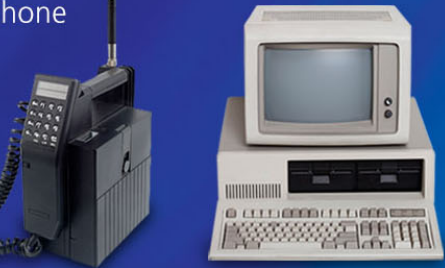

Why are you still using an AFM designed in the $80^{\prime}$ 's?
It is time to upgrade your AFM

Minimum $\$ 20,000$ trade-in discount for purchases before August 31st

Asylum Research is today's technology leader in AFM 


\title{
Ultraviolet laser deposition of graphene thin films without catalytic layers
}

\author{
S. R. Sarath Kumar and H. N. Alshareef ${ }^{a)}$ \\ Materials Science and Engineering, King Abdullah University of Science and Technology (KAUST), \\ Thuwal 23955-6900, Saudi Arabia
}

(Received 9 September 2012; accepted 17 December 2012; published online 9 January 2013)

\begin{abstract}
In this letter, the formation of nanostructured graphene by ultraviolet laser ablation of a highly ordered pyrolytic graphite target under optimized conditions is demonstrated, without a catalytic layer, and a model for the growth process is proposed. Previously, graphene film deposition by lowenergy laser $(2.3 \mathrm{eV})$ was explained by photo-thermal models, which implied that graphene films cannot be deposited by laser energies higher than the C-C bond energy in highly ordered pyrolytic graphite $(3.7 \mathrm{eV})$. Here, we show that nanostructured graphene films can in fact be deposited using ultraviolet laser $(5 \mathrm{eV})$ directly over different substrates, without a catalytic layer. The formation of graphene is explained by bond-breaking assisted by photoelectronic excitation leading to formation of carbon clusters at the target and annealing out of defects at the substrate. (C) 2013 American Institute of Physics. [http://dx.doi.org/10.1063/1.4773987]
\end{abstract}

Graphene, with its planar $s p^{2}$ bonded honeycomb structure, exhibits unique electrical properties that make it a preferred material for next generation electronics. ${ }^{1,2}$ Together with the peculiar nature of electron transport in graphene, described by the Dirac equation and scalability of graphenebased devices, the chemical and mechanical stability of graphene offers added advantage to device engineers. With the advent of reliable synthesis routes for high quality graphene, many thought experiments involving quantum mechanics could be implemented experimentally. Different techniques exist for the fabrication of graphene, each having its own advantages. ${ }^{3,4}$ For instance, mechanical exfoliation is a simple top-down approach that does not require high process temperatures. However, the process is labor-intensive while non-uniformity and presence of residues that limit the mobility of the exfoliated layers are serious drawbacks. High temperature processes are required to remove the residues. ${ }^{4}$ Epitaxial growth on $\mathrm{SiC}^{5}$ by the sublimation of $\mathrm{Si}$ atoms at high temperatures and high vacuum or by etching ${ }^{6}$ on the other hand leads to better quality of graphene, but involves high costs in synthesis and subsequent transferring ${ }^{7}$ of graphene to desired substrates. ${ }^{8}$ Uniform epitaxial graphene layers can be deposited over large areas covered with catalytic metal layers, such as $\mathrm{Ni}, \mathrm{Pt}, \mathrm{Ru}, \mathrm{Ir}, \mathrm{Cu}$, and $\mathrm{Co}$, or metal carbides by chemical vapor deposition ${ }^{9,10}$ of hydrocarbons as well as pulsed laser deposition ${ }^{11}$ (PLD) using suitable targets, such as highly ordered pyrolytic graphite (HOPG). Here again, removal of the undesired catalysts ${ }^{12}$ and transferring of graphene to suitable substrates limit the range of applicability. Wet chemical methods are easy up-scalable graphene synthesis routes, but marred by the quality of graphene since the resultant material after chemical reduction contains graphene oxide, which is detrimental to the device performance.

Direct synthesis of graphene on different substrates of practical importance without the need of a catalyst or a mechanical route is highly desirable for integrating graphene

\footnotetext{
${ }^{\text {a) }}$ Author to whom correspondence should be addressed. Electronic mail: husam.alshareef@kaust.edu.sa.
}

into existing technologies. Nanostructured graphene structures have been observed in carbon films grown at $900^{\circ} \mathrm{C}$ using PLD from HOPG target using a Nd:YAG laser operating at $532^{13}$ and $1064 \mathrm{~nm} .{ }^{14}$ Free-standing graphene has been obtained recently by Qian et al. ${ }^{15}$ using PLD employing a $\mathrm{Nd}$ :YAG laser (wavelength $=532 \mathrm{~nm}$ ). Since the C-C bond energy of $\mathrm{HOPG}$ is $3.7 \mathrm{eV}$, exfoliation from HOPG graphene using a photon energy of $2.3 \mathrm{eV}$ (corresponding to $532 \mathrm{~nm}$ ) is explained by a photo-thermal mechanism that involves no bond breaking. If the photo-thermal mechanism is indeed true, it would imply that graphene films may not be formed by lasers having photon energies in excess of $3.7 \mathrm{eV}$ since the probability that all the bonds of HOPG will be broken is quite high and hence the deposit will be predominantly amorphous carbon.

In order to check if graphene can be deposited using a laser with energy in excess of the $\mathrm{C}-\mathrm{C}$ bond energy of HOPG, without the need for a sacrificial catalytic layer, we have employed a $\mathrm{KrF}$ laser (wavelength $=248 \mathrm{~nm}$, corresponding to a photon energy of $5 \mathrm{eV}$ ) and films were deposited directly over different substrates. In this Letter, we report the synthesis of graphene films using $\mathrm{KrF}$ laser and offer a model for the growth process involved.

Graphene films were deposited by pulsed laser ablation of a HOPG target (SPI Supplies, $444 \mathrm{HP}-\mathrm{AB}$ ) using a $\mathrm{KrF}$ excimer laser (20 ns pulse width and $10 \mathrm{~Hz}$ repetition rate). Films were deposited on $\mathrm{SiO}_{2} / \mathrm{Si}, \mathrm{SiN}_{\mathrm{x}} / \mathrm{Si}$, and p-Si substrates, which were cleaned ultrasonically in de-ionized water and isopropyl alcohol and jet-dried using high purity $(5 \mathrm{~N})$ nitrogen gas. The deposition chamber was evacuated to a base pressure of less than $10^{-8}$ mbar and the films were grown in $20 \mathrm{mTorr}$ of high purity $(5 \mathrm{~N})$ argon gas allowed into the chamber using a mass flow controller. Films were also grown in 20 mTorr of oxygen. PLD was carried out at three different substrate temperatures $(300,593$, and $973 \mathrm{~K})$ and laser fluences $(3,5$, and $6 \mathrm{~J} \mathrm{~cm}^{-2}$ ). The rotating target carousel ensured uniform ablation while the rotating substrate holder ensured uniform deposition of the films. Graphene films were analyzed using X-ray diffraction (XRD, D8 Discover, Bruker), micro-Raman spectroscopy (LabRAM ARAMIS, Horiba-Jobin Yvon), and 




FIG. 1. Raman spectra of graphene films deposited at $973 \mathrm{~K}$ with different laser fluences.

scanning electron microscopy (SEM, Nova NanoSEM 630 FESEM, FEI). Micro-Raman spectra were acquired with notch filters cutting at $\sim 100 \mathrm{~cm}^{-1}$, at a spectral resolution of $0.5 \mathrm{~cm}^{-1}$ after exciting the films with a HeNe laser $(633 \mathrm{~nm}$ and $17 \mathrm{~mW}$ at source). The spectra were analyzed using a peak fitting procedure to extract the peak positions, linewidths (FWHM), and peak intensities. The electrical conductivity of the films was measured in the temperature range 300-500 K by using a commercial setup (RZ2001i, Ozawa Science) employing a linear four-probe method.

Figure 1 shows the normalized Raman spectra of graphene films deposited at $973 \mathrm{~K}$ using three different laser fluences. The $\mathrm{G}$ band (around $1600 \mathrm{~cm}^{-1}$ ) is due to first order Raman scattering involving the in-plane $\mathrm{E}_{2 \mathrm{~g}}$ phonon at the center of the Brillouin zone. ${ }^{5}$ The presence of $\mathrm{G}$ band is considered a signature of formation of hexagonal graphitic lattice. ${ }^{16}$ The $\mathrm{D}$ band appearing at $1320 \mathrm{~cm}^{-1}$ is due to the zoneboundary phonons, corresponding to the disorder in the twodimensional graphitic network. ${ }^{17}$ The high intensity of the D band can be associated to the non-sp $p^{2}$ bonds formed at the edges of nano-structured graphene. The ratio of intensity of $\mathrm{D}$ band to $G$ band $\left(\mathrm{I}_{\mathrm{D}} / \mathrm{I}_{\mathrm{G}}\right)$ is a measure of the amount of defects of graphene. $\mathrm{I}_{\mathrm{D}} / \mathrm{I}_{\mathrm{G}}$ of films is found to increase with increasing laser fluence (see Table I). The higher defect concentration in films grown at higher laser fluence can be attributed to the impingement of more energetic species at the substrate during laser ablation. Additionally, a combination of the $\mathrm{D}$ and $\mathrm{G}$ bands (referred to as the S3 band) appears as a separate band at $\sim 2910 \mathrm{~cm}^{-1}$. The symmetric 2D (sometimes referred to as $\mathrm{D}^{\prime}$ ) band at $\sim 2625 \mathrm{~cm}^{-1}$ is a characteristic feature of graphene, arising from second order zone-boundary phonons. ${ }^{18}$ The 2D band will be present even in the absence of defects and becomes asymmetric with the appearance of a lower wavenumber shoulder peak, when the number of graphene layers increases beyond 5, as in the case of graphite. ${ }^{11}$ The ratio of intensity of $2 \mathrm{D}$ band to $\mathrm{G}$ band $\left(\mathrm{I}_{2 \mathrm{D}} / \mathrm{I}_{\mathrm{G}}\right)$ is often used to extract the number of layers of graphene. ${ }^{5,18} \mathrm{I}_{2 \mathrm{D}} / \mathrm{I}_{\mathrm{G}}$ is 2 for monolayer graphene and decreases with increase in number of layers, since the $\mathrm{G}$ band becomes more prominent. The FWHM of the 2D band for the film deposited at $6 \mathrm{~J} \mathrm{~cm}^{-2}$ is $95 \mathrm{~cm}^{-1}$, which is higher than $74 \mathrm{~cm}^{-1}$, reported for trilayer graphene. ${ }^{8}$ The FWHM of the 2D peak is considered an unambiguous fingerprint in identifying the number of graphene layers, since it increases with the number of graphene layers. Taking these into consideration and also from Fig. 1 and Table I, it is clear that the film deposited at $6 \mathrm{~J} \mathrm{~cm}^{-2}$ shows features of few-layer graphene with 3-4 layers while films grown at lower laser fluences show features of multilayer graphene. Though direct transmission electron microscopy images may provide additional visual information on the graphene films, well-established Raman analysis was used for comparing the quality and number of layers in our films. The Raman technique is most appropriate in this case since the laser beam diameter is a few $\mu \mathrm{m}$ and can provide more representative information on our in-plane grown films. Raman spectra obtained for films grown on different substrates under similar conditions were identical, indicating that graphene film can be deposited independently of the substrates used. Also, films grown under 20 mTorr of oxygen showed no peaks corresponding to graphene, due to oxidation of the films to graphitic oxide.

The SEM images of the films revealed a smooth featureless surface indicating uniform coverage of the graphene films. The surface of the films was lightly scratched to observe the graphene layers in a wrinkled form, as shown in Fig. 2(a). The inset to Fig. 2(a) shows a delaminated graphene sheet from the scratched region. The SEM images indicate that the graphene layers grow perpendicular to the c-axis. Grazing incidence X-ray diffraction measurements of the films revealed no peaks while the conventional XRD (Bragg-Brentano geometry) yielded a broad peak, also suggesting the in-plane growth of the films. The XRD pattern of the films was identical and is shown in Fig. 2(b). The broad peak around $20^{\circ}-30^{\circ}$ corresponds to $\mathrm{C}$ (200) from the graphene layer. ${ }^{19}$ From the large FWHM of the (200) peak, it is evident that the graphene films are nanostructured and there exists only a short range order in the stacked sheets. Moreover, the absence of a sharp peak corresponding to graphite around $26^{\circ}$ indicates that the number of layers of the graphene films is indeed low.

The electrical resistivity of the films is found to decrease with decreasing laser fluence, presumably owing to the lower density of defects, as shown in Fig. 3. The resistivity falls with increase in temperature, indicating semiconducting nature of the films. The electrical resistivity of the films is

TABLE I. Peak intensities and ratios for D, G, and 2D bands for films deposited at $973 \mathrm{~K}$ with different laser fluences.

\begin{tabular}{|c|c|c|c|c|c|c|c|c|}
\hline $\begin{array}{l}\text { Laser fluence } \\
\left(\mathrm{J} \mathrm{cm}^{-2}\right)\end{array}$ & $\begin{array}{l}\text { No. of } \\
\text { shots }\end{array}$ & $\begin{array}{l}\text { Thickness } \\
\text { of film (nm) }\end{array}$ & $\begin{array}{l}\text { ID } \\
\text { (a.u.) }\end{array}$ & $\begin{array}{c}\mathrm{IG} \\
\text { (a.u.) }\end{array}$ & $\begin{array}{l}\text { I2D } \\
\text { (a.u.) }\end{array}$ & $\mathrm{ID} / \mathrm{IG}$ & $\mathrm{I} 2 \mathrm{D} / \mathrm{IG}$ & $\begin{array}{l}\text { Electrical resistivity } \\
\qquad(\mathrm{m} \Omega \mathrm{cm})\end{array}$ \\
\hline 3 & 1000 & 11 & 2653 & 1544 & 231 & 1.72 & 0.15 & 14.7 \\
\hline 4 & 1000 & 16 & 2814 & 1405 & 250 & 2.00 & 0.18 & 17.8 \\
\hline 6 & 1000 & 30 & 5572 & 2537 & 733 & 2.20 & 0.29 & 19.2 \\
\hline
\end{tabular}



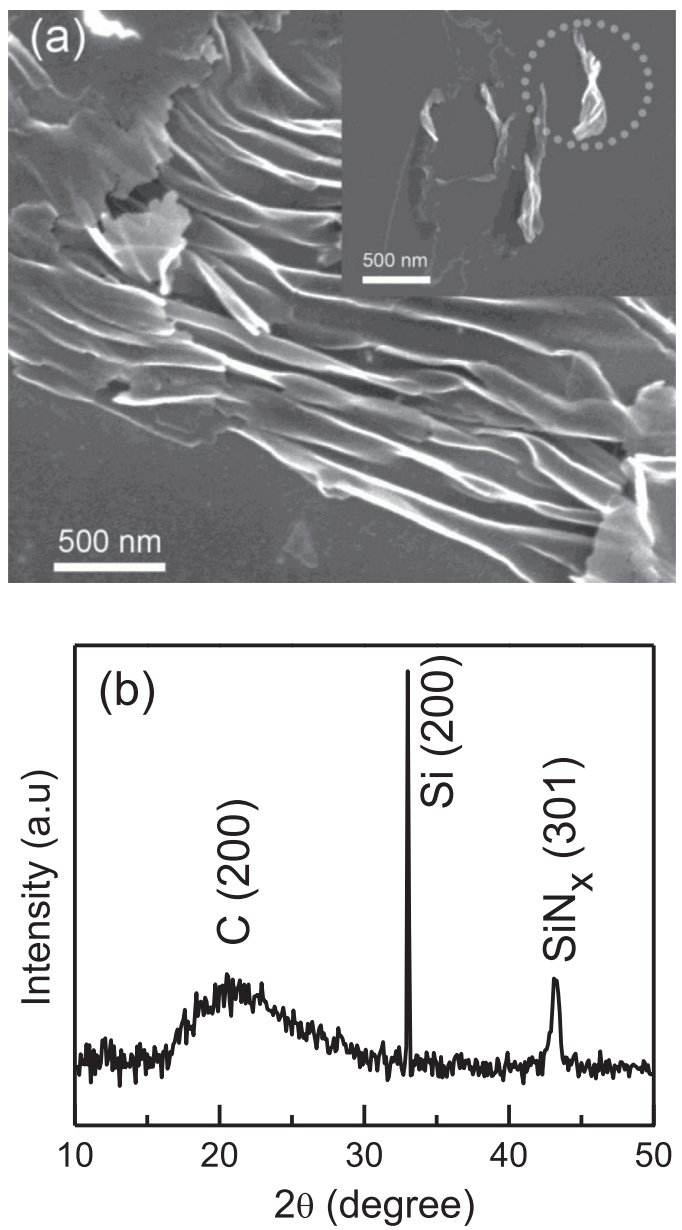

FIG. 2. (a) SEM image of the surface of graphene film. The wrinkled structure is obtained by scratching the surface lightly with a knife edge. Inset shows a delaminated graphene sheet (inside the dotted circle) from another scratched region. The otherwise smooth featureless surface is also visible. (b) XRD pattern of the graphene films.

lower compared to that reported ${ }^{20}$ for chemical vapor deposited graphene films, but higher than that reported ${ }^{10}$ for isolated and suspended monolayer graphene, which is not surprising since our graphene films are nanostructured and contains more number of layers and defects. The lower electrical resistivity of the films makes them ideal for various electronic applications.

Keeping the results in mind, we propose a qualitative model for the formation of graphene by PLD. Earlier

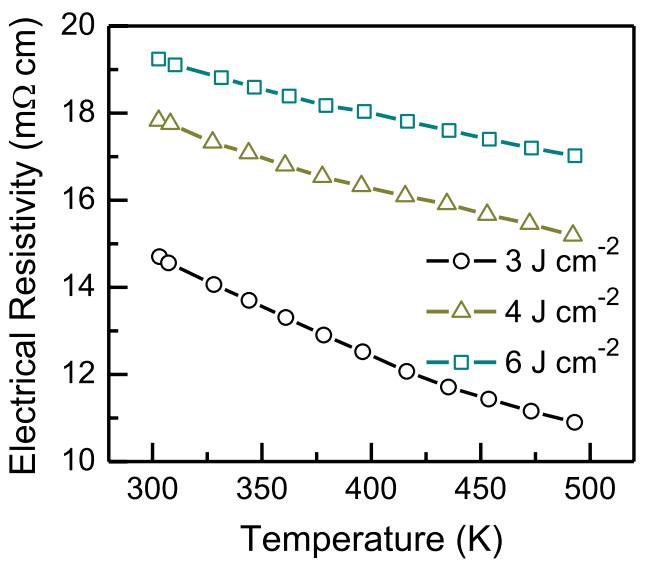

FIG. 3. Electrical resistivity as a function of temperature for graphene films deposited at three laser fluences. attempts on graphene synthesis by PLD from compressed graphite pellets and other carbon sources had been failures. ${ }^{15}$ The target (HOPG) has a layered structure, which is crucial for the formation of graphene. With each laser shot at $5 \mathrm{eV}$, the graphitic bonds of HOPG are rapidly broken by photoelectronic excitation, ${ }^{21}$ resulting in the ablation of nanostructured carbon species. At lower laser fluences, the volume of ablated carbon species with each laser shot is lower, as evident from the decrease in film thickness. Also, larger carbon cluster ions are ejected from the target as the laser fluence decreases, as has been reported by previous mass spectroscopy studies. ${ }^{21}$ The low volume of larger clusters striking the substrate per laser shot at lower kinetic energies favors the nucleation of the carbon species to form nano-crystalline graphene, with less defects and more number of layers (greater than 5, for the films deposited with lower laser fluences), though the overall film thickness is lower. Moreover, at lower fluences, re-sputtering of the deposited film by the impinging ablated species will be lower, which also reduces the defect concentration. The high substrate temperature also helps in the annealing of the defects and promotes the layered growth.

The role of high substrate temperature in the formation of graphene films is evident from Fig. 4 where the Raman spectra for films deposited at three different substrate temperatures is shown. The distinctive D and G bands of graphene films gradually disappear as the substrate temperature is lowered. Apart from being significantly broadened, the D band shows a positive Raman shift while the $G$ band shows a negative shift with decreasing substrate temperature. The broadening of the bands is attributed to the disordered amorphous $s p^{2}$ configuration. ${ }^{22}$ The features clearly indicate that the quality of graphene films drops significantly with lower substrate temperatures. It is clear that at lower substrate temperature, the ablated carbon clusters cannot coalesce to form graphene sheets at the substrate. The electrical resistivity of the films grown at lower substrate temperatures was too high to be measured by the four-probe method, a direct consequence of the loss of the crystalline $s p^{2}$ networks. Similarly, the films grown under 20 mTorr of oxygen, irrespective of the substrate temperatures and laser energies were also highly resistive, due to the formation of graphitic oxide.

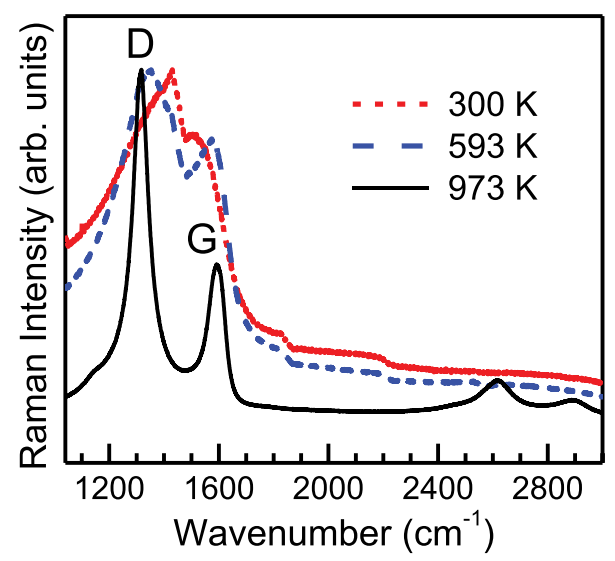

FIG. 4. Raman spectra obtained from films formed at different substrate temperatures. 
In summary, we have deposited nanostructured graphene films directly over different substrates under optimized conditions without the need for a catalytic layer, using PLD employing a $\mathrm{KrF}$ excimer laser. With increase in laser fluence, the number of layers of graphene decreased while defects increased. The films were semiconducting in nature with the electrical resistivity decreasing with decreasing defect concentration. The formation of graphene films is attributed to the ejection of carbon clusters by the breaking of HOPG bonds through photoelectronic excitation and the subsequent deposition over the substrate kept at a high temperature, which help to anneal the defects.

\footnotetext{
${ }^{1}$ A. K. Geim and K. S. Novoselov, Nature Mater. 6(3), 183-191 (2007).

${ }^{2}$ A. K. Geim, Science 324(5934), 1530-1534 (2009).

${ }^{3}$ C. Soldano, A. Mahmood, and E. Dujardin, Carbon 48(8), 2127-2150 (2010).

${ }^{4}$ W. Choi, I. Lahiri, R. Seelaboyina, and Y. S. Kang, CRC Crit. Rev. Solid State Mater. Sci. 35(1), 52-71 (2010).

${ }^{5}$ C. Faugeras, A. Nerriere, M. Potemski, A. Mahmood, E. Dujardin, C. Berger, and W. A. de Heer, Appl. Phys. Lett. 92(1), 011914 (2008).

${ }^{6}$ S. Raghavan, T. J. Denig, T. C. Nelson, and C. D. Stinespring, J. Vac. Sci. Technol. B 30(3), 030605 (2012).

${ }^{7}$ X. Li, Y. Zhu, W. Cai, M. Borysiak, B. Han, D. Chen, R. D. Piner, L. Colombo, and R. S. Ruoff, Nano Lett. 9(12), 4359-4363 (2009).

${ }^{8}$ D. S. Lee, C. Riedl, B. Krauss, K. von Klitzing, U. Starke, and J. H. Smet, Nano Lett. 8(12), 4320-4325 (2008).
}

${ }^{9}$ A. Reina, X. Jia, J. Ho, D. Nezich, H. Son, V. Bulovic, M. S. Dresselhaus, and J. Kong, Nano Lett. 9(1), 30-35 (2009).

${ }^{10}$ K. S. Kim, Y. Zhao, H. Jang, S. Y. Lee, J. M. Kim, K. S. Kim, J.-H. Ahn, P. Kim, J.-Y. Choi, and B. H. Hong, Nature 457(7230), 706-710 (2009).

${ }^{11}$ A. T. T. Koh, Y. M. Foong, and D. H. C. Chua, Appl. Phys. Lett. 97(11), 114102-114103 (2010).

${ }^{12}$ A. J. Pollard, R. R. Nair, S. N. Sabki, C. R. Staddon, L. M. A. Perdigao, C. H. Hsu, J. M. Garfitt, S. Gangopadhyay, H. F. Gleeson, A. K. Geim, and P. H. Beton, J. Phys. Chem. C 113(38), 16565-16567 (2009).

${ }^{13}$ C. Scilletta, M. Servidori, S. Orlando, E. Cappelli, L. Barba, and P. Ascarelli, Appl. Surf. Sci. 252(13), 4877-4881 (2006).

${ }^{14}$ E. Cappelli, S. Orlando, M. Servidori, and C. Scilletta, Appl. Surf. Sci. 254(4), 1273-1278 (2007).

${ }^{15}$ M. Qian, Y. S. Zhou, Y. Gao, J. B. Park, T. Feng, S. M. Huang, Z. Sun, L. Jiang, and Y. F. Lu, Appl. Phys. Lett. 98(17), 173108 (2011).

${ }^{16}$ L. M. Malard, M. A. Pimenta, G. Dresselhaus, and M. S. Dresselhaus, Phys. Rep. 473(5-6), 51-87 (2009).

${ }^{17}$ D. Graf, F. Molitor, K. Ensslin, C. Stampfer, A. Jungen, C. Hierold, and L. Wirtz, Nano Lett. 7(2), 238-242 (2007).

${ }^{18}$ A. C. Ferrari, J. C. Meyer, V. Scardaci, C. Casiraghi, M. Lazzeri, F. Mauri, S. Piscanec, D. Jiang, K. S. Novoselov, S. Roth, and A. K. Geim, Phys. Rev. Lett. 97(18), 187401 (2006).

${ }^{19}$ Z. Ji, X. Shen, Y. Song, and G. Zhu, Mater. Sci. Eng., B 176(9), 711-715 (2011).

${ }^{20}$ I. Vlassiouk, S. N. Smirnov, I. Ivanov, P. F. Fulvio, S. Dai, H. Meyer, M. F. Chi, P. Datskos, and N. V. Lavrik, Nanotechnology 22(27), 275716 (2011).

${ }^{21}$ J. J. Gaumet, A. Wakisaka, Y. Shimizu, and Y. Tamori, J. Chem. Soc., Faraday Trans. 89(11), 1667-1670 (1993).

${ }^{22}$ A. C. Ferrari and J. Robertson, Phys. Rev. B 61(20), 14095-14107 (2000). 